\title{
LA FORMACIÓN ESPIRITUAL CENTRO VITAL UNIFICADOR DE LA FORMACIÓN SACERDOTAL (PDV 45)
}

\section{Un proyecto de formación espiritual en la etapa de estudios eclesiásticos}

LUÍS RUBIO MORÁN

DiRECTOR ESPIRITUAL dEL SEMINARIO MAYOR

ÉVORA, PORTUGAL

DOI: https://doi.org/10.52039/seminarios.v54i189-190.521

Introducción (51); I. Condicionamientos de la centralidad de la formación espiritual en el proyecto del seminario (52); II. La identidad del presbítero, objetivo de la formación espiritual (57); 2.1. Una identidad específica (57); 2.2. La pastoreitas como núcleo identificador (58); 2.3. Aspectos fundamentales de la pastoreitas, objetivos de la formación espiritual del presbítero (59); III. Medios y actividades para la formación espiritual (63); 3.1. Formación teórica: Identidad-espiritualidad del presbítero diocesano secular (65); 3.2. Formación práctica: Medios y actividades (66); 3.2.1. La comunidad educativa (66); 3.2.2. Las formas de expresión (69); 3.2.3. Las experiencias fundantes (72); 3.2.3.1. Experiencias fundantes existenciales (72); 3.2.3.2. Experiencias fundantes celebrativas (73); 3.2.4. La atención al viandante (PDV 61) (74); Anexo: Guía para las entrevistas con el director espiritual (78). 


\section{INTRODUCCIÓN}

El título de esta aportación a este Simposio recoge una expresión de la PDV en el n. 45 que a la letra suena así: así como para todo fiel la formación espiritual debe ser central y unificadora en su ser y en su vida de cristiano, ...de la misma manera, para todo presbítero la formación espiritual constituye el centro vital que unifica y vivifica su ser sacerdote y su ejercer el sacerdocio.

La expresión se ha hecho ya habitual, casi diría que tópica, al hablar de la formación presbiteral. Con ella se quiere subrayar la trascendencia de este aspecto de la formación, como la misma Exhortación glosa a renglón seguido, citando una de las Proposiciones del Sínodo: la formación espiritual constituye un elemento de máxima importancia en la educación sacerdotal.

El tema podría orientarse, o bien de manera demostrativa, proponiendo argumentos y motivos de esa centralidad vital y unificadora, o bien intentando mostrar cómo en la realidad concreta de la preparación para el ministerio presbiteral la formación espiritual vitaliza y unifica las otras áreas formativas. Esta segunda orientación resulta más oportuna porque puede ofrecer sugerencias para la práctica. Y parece más necesaria, dado que en los Documentos y entre los tratadistas encontramos habitualmente adecuadas y hermosas formulaciones teóricas pero sin apenas orientaciones o directrices para convertir en realidad las solemnes afirmaciones.

Personalmente he preferido situarme en esta segunda perspectiva, por lo que añadí el subtítulo, con la osada pretensión nada menos que de ofrecer un Proyecto de formación espiritual en la etapa de estudios eclesiásticos. No pretendo presentar, como es obvio, un modelo para ser imitado, sino un simple ejemplo de proyecto experimentado ya durante algunos años en el seminario de Evora (Portugal), un seminario pequeño, con unas características peculiares ${ }^{1}$, pero que por su número se puede con-

1 El seminario está formado en este momento por 20 alumnos procedentes de las dos diócesis del sur de Portugal, Evora y Faro, de la diócesis de Mindelo, en Cabo Verde, y de dos ucranianos. El proyecto que se presenta ha sido elaborado a lo largo de varios años, durante los cuales existía un grupo numeroso de seminaristas venidos de Timor occidental, en Indochina, antigua colonia portuguesa, y de otro grupo significativo de seminaristas de la diócesis de Ondjiva, en Angola, junto con el grupo de los de Cabo Verde. La característica intercontinental e internacional, pues, ha estado presente siempre en la elaboración y adaptación del proyecto educativo. 
siderar como un tipo frecuente entre nosotros. Espero que de esta manera pueda servir en alguna medida a las inquietudes de los componentes de este Simposio, ofreciéndoles algunas pistas, tanto teóricas como prácticas, que puedan orientarles para hacer efectiva en sus propios ambientes esa confesada centralidad vital unificadora de la formación espiritual ${ }^{2}$.

\section{CONDICIONAMIENTOS DE LA CENTRALIDAD DE LA FORMACIÓN ESPIRITUAL EN EL PROYECTO DEL SEMINARIO}

La centralidad de la formación espiritual se halla condicionada, cuando se trata de plasmarla en un proyecto educativo, por múltiples factores.

2 La elaboración de este trabajo, y, en especial, la del Proyecto educativo, tiene su fundamento en numerosos documentos y estudios. Ofrezco aquí solo los más significativos, evitando citaciones particulares en el resto del trabajo. Solo en algunas ocasiones, y para aspectos concretos, incluiré referencias a estos u otros trabajos específicos. Decretos conciliares Presbyterorum ordinis y Optatam totius; Sagrada Congregación para la Educación católica: Ratio fundamentalis institutionis sacerdotalis $(1970,1976)$, Directrices para la formación de los formadores(1993, texto en SEMINARIOS, n. 132, pp. 221254); Juan Pablo II, Exhortación Apostólica Pastores dabo vobis (PDV); Conferencia episcopal española: Plan de formación sacerdotal para los seminarios mayores (1996); Conferencia episcopal portuguesa: Notas fundamentais de formação sacerdotal (1976); Conferenza episcopale italiana: La formazione dei presbityeri nella Chiesa italiana (2006). AA.VV., Pastores dabo vobis. Texto y Comentario, SEMINARIOS, nn.125-126(1992), especialmente los estudios de J. Cabezas, La identidad del presbítero(pp.309-318), de L. Rubio Morán, La formación del pastor a la luz de Pastores dabo vobis.Una lectura global y unitaria de la Exhortación (p.333-358); J.Rico-C.R. López, El proceso educativo de la formación sacerdotal, (pp.375-400); AA.VV., Formar presbíteros hoy. Estudios sobre el nuevo Plan de formación sacerdotal para los seminarios mayores de España, SEMINARIOS, nn.107-108 (1988), especialmente los estudios: L. Rubio Morán, El ministerio presbiteral: perspectivas y acentos teológicos,(pp. 17-42); J. García, La formación espiritual, (pp.105118); A. Montalvo, El plan de formación: un proyecto pedagógico, (pp.165178); Comisión episcopal del Clero, La formación espiritual de los sacerdotes según Pastores dabo vobis, Edice, Madrid 1995; J. M. Imizcoz, Experiencia de Dios y formación vocacional, BAC, Madrid 2004; S. Gamarra, Manual de espiritualidad sacerdotal, Monte Carmelo, Burgos 2008; J. M. Uriarte, Ministerio presbiteral y espiritualidad, Idatz, San Sebastián, 1999. 
El primero de ellos es el hecho, constatado por todos y con frecuencia lamentado en las reuniones de formadores, de que en la organización de los seminarios la formación intelectual se convierte en la práctica en un elemento altamente condicionante. A él, en efecto, se le dedica la mayor y mejor parte del tiempo, y es sabido, como afirmara el Cardenal Martini hablando de la oración en la espiritualidad del sacerdote 3 , que la importancia de algo se muestra en el tiempo que se le dedica, en el lugar en que se lo sitúa. No parece sino que la afirmación conciliar, acogida y repetida abundantemente en los documentos posteriores, tanto pontificios como episcopales, de que la finalidad del seminario es formar pastores (OT 4) no ha conseguido desplazar en tiempo, interés y energías, a la de formar doctores, maestros, que puedan ser transmisores de la inteligencia de la fe, del depósito de la doctrina cristiana4.

Una segunda presión procede de la comprensión misma de lo espiritual. Es bien sabido que durante mucho tiempo, y hasta tiempos no tan lejanos, la formación espiritual tenía como objetivo la configuración de un sacerdote-sacerdote, caracterizado por su piedad y devoción, dispuesto y preparado para su función fundamental de garantizar, alimentar y cuidar la piedad del pueblo cristiano. Así se concibió y llevó a cabo con éxito, a partir del Informe Vico5, la reforma de los aseglarados seminarios españoles en toda la mitad del siglo XX. La reforma que se llevó a cabo con enorme esfuerzo y tesón, y con mayor o menor éxito en todos los seminarios españoles, especialmente en los que se fueron encomendando a la Hermandad de Sacerdotes Operarios, conforma un tipo de seminario que se puede calificar como el seminario disciplinario y piadoso ${ }^{6}$, donde

3 Cfr. El ejercicio del ministerio, fuente de espiritualidad sacerdotal, en Congreso de espiritualidad sacerdotal, Edice Madrid 1989, especialmente pp.185-189.

4 No se podrá sin duda urgir el dato, pero mientras que la formación intelectual ocupa 6 amplios números en la Pastores dabo vobis, a la formación pastoral solo se le dedican 3; a la formación humana 2 y 5 a la formación espiritual.

5 En Informe Vico es de 1891-92. El texto del Informe fue publicado integro en traducción española, y gracias a la amabilidad del investigador Vicente Cárcel Ortí, que lo descubrió en el Archivo Vaticano, en SEMINARIOS, 26(1980)277-432.

6 Cfr. Mi estudio sobre Cien años de seminarios en España, en Estudios, seminarios y pastoral en un siglo de la historia en España (1892-1992), Pontificio Colegio español de S. José, Primer centenario, Roma 1992, pp.7095, especialmente sobre la piedad, pp.85-88, la cita en p. 85. 
las prácticas de piedad ocupaban casi tanto tiempo como el estudio.

Era común la convicción expresada por uno de los más ilustres formadores de seminarios de los comienzos del siglo XX, quien, al ser nombrado Obispo de Orense lo primero que hace es promulgar un Reglamento para su seminario, que se convirtió en modelo para la mayor parte de los de España, que parte de esta afirmación: cuando la piedad entra en el seminario los estudios mejoran, la disciplina se vigoriza, el orden aumenta, reina la paz... y cual brisa suave descendida del cielo orea los corazones juveniles y los alienta "ad omne opus bonum". Es lo que ha dicho el Espíritu Santo: "pietas utilis ad omnia"7. En coherencia perfecta con esta perspectiva se señala como función del director espiritual formar el corazón de los alumnos en el espíritu eclesiástico, cultivando en sus almas aquellas virtudes de que se nutre la sólida piedad, fundamento de la vida sacerdotal8 .

Aunque este modelo de seminario ya no existe entre nosotros, superado a raíz del informe Tedeschini ${ }^{9}$ y del celo apostólico provocado por la guerra civil10, por el seminario calificado como apostólico", y, superado sobre todo por los variados intentos del seminario pastoral, fruto del Concilio, aun en gestación ${ }^{11}$, no dejan de advertirse a veces ciertas nostalgias del mismo cuando parece valorarse sobremanera el número y variedad de actos o prácticas, aunque ahora se trate ya de las litúrgicas, o cuando a propósito del discernimiento vocacional parece ponerse el acento en las manifestaciones religiosas.

Una tercera presión o condicionante se encuentra también cuando la formación espiritual se considera como un aspecto o un elemento más al lado de los otros. La persona aparece así, quiérase o no, como dividida en partes, en fragmentos, de los que uno es el más importante o central y los otros deben girar a su alrededor, o a lo sumo recibir su influencia, pero permaneciendo cada uno independiente y autónomo en sí mismo. Esta perspectiva cambia cuando se la considera no como aspecto, elemento o parte sino como dimensión, algo que invade y atraviesa y per-

7 Mons. Ilundain, en la Introducción al Reglamento del Seminario de Orense(1906), pp. 8-9.

8 ld. Ibíd. art. 30.

9 El texto, descubierto también en el Archivo Vaticano por el mismo V. Cárcel Ortí, ha sido publicado por el Pontificio Colegio Español y Ediciones Sígueme, bajo el título: Informe de la Visita apostólica a los seminarios españoles en 1933-34, Roma-Salamanca 2006.

10 Cfr. L. Rubio Morán, o. c. pp. 95-124, y, sobre todo, 104-116.

11 ld. Ibíd. 140-151. 
meabiliza toda la realidad de la persona, y que, por lo mismo, afecta a toda su realidad, a todo su ser y sus actividades

El condicionamiento fundamental, si bien en este caso totalmente positivo, es la nueva consideración de la vida espiritual, de la espiritualidad en general, y, por lo mismo, de la espiritualidad presbiteral.

La vida espiritual, en efecto, se concibe hoy como la vida en el Espíritu, como el vivir concreto de cada día, en todas sus manifestaciones, sumergidos en el Espíritu de Jesús, orientando pensamientos, sentimientos y conductas bajo la acción e inspiración del Espíritu Santo, o dicho de otra manera, persona espiritual es aquella que vive su existencia integrada desde el valor religioso y en concreto desde la vida teologal.

Esta es la perspectiva que nos ofrece, por ejemplo, S. Gamarra en su reciente y valiosa obra Manual de Espiritualidad sacerdotal. La espiritualidad designa la estructuración de la persona cristiana (en nuestro caso del sacerdote) desde la fe o desde la vida teologal, teniendo, por tanto, como elementos básicos, la vida en Cristo, el ser en Cristo, el "ser y vivir en la iglesia", el "compromiso por el hermano12. Se trata, por tanto, de vivir la identidad cristiana en todo su ser, de que nada de la persona humana quede fuera del ser y vivir en Cristo, que en El están presentes todas las dimensiones de la persona, también la inserción en el mundo y las relaciones con los demás, lo que requiere, por una parte, la comprensión de la vida cristiana en sus elementos más básicos y radicales.., porque la estructuración de la persona cristiana va a depender de la visión que tenga de lo que es ser cristiano, y por otra, la atención al proceso del cristiano, de su integración como persona cristiana, lo que supone formación, acompañamiento. Todo ello aplicado al sacerdote significa que hay una correspondencia plena entre identidad y espiritualidad ${ }^{13}$.

E. Bianchi, el monje laico tan estimado hoy en muchos ambientes sacerdotales, nos hablará de la vida espiritual como la experiencia de Dios, la vida de relación con Dios, una relación que ha de ser vivida en un contexto comunitario y que se basa en la escucha de la palabra de Dios contenida en las Escrituras, plasmada por la eucaristía y articulada en una vida de fe, de esperanza y de caridad ${ }^{14}$. La experiencia de Dios se realiza en la fe, y puede decirse que consiste fundamentalmente en la experiencia de ser precedidos, en la experiencia de ser hijos en el Hijo, cau-

12 S. Gamarra, Manual de espiritualidad sacerdotal, Monte Carmelo, Burgos 2008, pp.81-82.

13 O.c. $83-85$.

14 Palabras de la vida interior, Sígueme, Salamanca 2006, p.17 
sada por el Espíritu el Señor, lo que provoca en nosotros la crisis de la imagen que tenemos de nosotros mismos, la conversión, el momento en que se fractura el yo, no el real sino el ideal que nos hemos forjado y que pretendíamos alcanzar como realización obligada de nosotros mismos. Esto supone asimismo la honestidad hacia la realidad y la fidelidad a la realidad, es decir, la adhesión a la realidad puesto que es en la historia y en lo cotidiano, con los otros y no sin los otros, donde tiene lugar nuestro conocimiento de Dios y donde crece nuestra relación con E/15.

Esta es la perspectiva asumida en la PDV, tanto por lo que se refiere a la vida espiritual cristiana en general como por lo que dice respecto a la espiritualidad presbiteral. La exhortación, en efecto, afirmará la relación esencial entre identidad del presbítero y su espiritualidad (cfr. todo el c. III, La vida espiritual del sacerdote). La vida espiritual se define como relación y comunión con Dios, como obra del Espíritu y empeña a la persona en su totalidad; introduce en la comunión profunda con Jesucristo, buen Pastor; conduce a una sumisión de toda la vida al Espíritu en una actitud filial respecto al Padre y en una adhesión confiada a la Iglesia..., se arraiga en la experiencia de la cruz para poder llevar, en comunión profunda, a la plenitud del misterio pascual (PDV 45). Por eso afirmará también que la formación espiritual... requiere ser estructurada según los significados y características que derivan de la identidad del presbítero y su ministerio. Y es en este contexto en el que se encuentra la afirmación de que la formación espiritual es central y unificadora en su ser y en su vivir y hacer (PDV 45).

Comentando y glosando el texto de OT sobre la formación espiritual (cfr. OT 8) señalará como valores y exigencias fundamentales de la misma: vivir íntimamente unidos a Jesucristo; cultivar con El una verdadera y profunda "forma de amistad"; la búsqueda de Jesús, que se realizará mediante la meditación fiel de la palabra de Dios, la participación activa en los sagrados misterios de la Iglesia, en el servicio de la caridad a los más pequeños (PDV 46). Además de la búsqueda de Jesús la vida espiritual, y, por lo mismo, la formación espiritual comporta también buscar a Cristo en los hombres, la entrega a su servicio como seguidor y representante del Cristo Pastor, de su caridad pastoral, el don de sí mismo por amor en el que encuentra su lugar, y, por tanto, su formación, la educación de la obediencia, del celibato y de la pobreza (PDV 49, cfr. n. 50).

Esta es la perspectiva que ha recogido y explicitado el Plan de Formación

15 Id. o.c., 18-19. Véase J.M. Imízcoz, Experiencia de Dios y formación vocacional, BAC, Madrid 2004, especialmente, cc. III y VI, experiencia de Dios y experiencia cristiana de Dios, respectivamente. 
Sacerdotal para los Seminarios Mayores, de la Conferencia episcopal española de 1986 y que se mantiene íntegramente en la edición de 1996. Así cuando expone la finalidad de la formación sacerdotal especialmente en la parte dedicada a identidad y espiritualidad del presbitero diocesano secular, bajo los epígrafes, vocación, consagración y misión de los presbíteros, el ministerio presbiteral o sus funciones, las relaciones específicas de la identidad y espiritualidad del presbítero diocesano secular (nn. 20-46), que marcarán las líneas fundamentales y los objetivos y medios de la formación espiritual (nn.61-90) ${ }^{16}$.

\section{LA IDENTIDAD DEL PRESBÍTERO, OBJETIVO DE LA FORMACIÓN ESPIRITUAL}

Si la espiritualidad cristiana consiste en vivir la identidad cristiana, la espiritualidad del presbítero vendrá definida y determinada desde la identidad teológica del mismo, que la reflexión postconciliar ha clarificado amplia y profundamente y de la que la Pastores dabo vobis, recogiendo a su vez las determinaciones del Concilio en LG, PO y OT, nos ofrece una síntesis magistral a la vez que magisterial.

Descubrir, valorar, asimilar, mediante la reflexión madura y la aplicación práctica (PDV 57), y comenzar a plasmar en el vivir esa identidad será el objetivo absoluto del Proyecto educativo del seminario, objetivo que marcará rumbo y unificará todos los otros aspectos de la formación 17 .

\subsection{Una espiritualidad específica}

Es convicción común y puede decirse que cada vez más indiscutida que el presbítero posee una espiritualidad específica, que no necesita de

16 La tercera edición del Plan de formación para los seminarios de Italia (2006) ya coloca la dimensión espiritual en el primer lugar entre las dimensiones, lo que significa que la considera como prioritaria y bien puede comenzar con la afirmación de que constituye el corazón que unifica y vivifica la vida y formación de los futuros presbíteros (n. 80), y le dedica 10 números, dos más que a la formación teológica.

17 Así claramente el Plan de Formación español. La formación espiritual del seminario tiene esta finalidad específica: cultivar la espiritualidad del presbítero diocesano secular (n. 64), que el Plan entiende y deriva de la identidad propia del presbítero (cfr. nn. 20-46). Con mayor claridad, como sugiero más arriba, el Plan italiano, que sistematiza las indicaciones de la Pastores dabo vobis (cfr. nn. 80-89) colocando en el centro de la formación espiritual la "caridad pastoral" que caracteriza y unifica su vida y su espiritualidad (n.84). 
otras espiritualidades, aunque no se excluya que algunas puedan ayudarle en esta especificidad: una espiritualidad que deriva de su identidad propia como christifidelis presbyter. Esta espiritualidad, enraizada, sin duda, como toda espiritualidad cristiana, en el bautismo, trae su origen del sacramento del orden, que lo conforma ontológicamente con Cristo Buen Pastor de la Iglesia. Esta espiritualidad

tiene un perfil específico que impregna y colorea toda la vida de los presbiteros. Es una espiritualidad diferente... Entre la teología del ministerio presbiteral y la espiritualidad del presbitero tiene que existir una sintonía. Dicho en formulación negativa, no es adecuada para el presbítero cualquier espiritualidad por recia y evangélica que sea. $A$ veces determinados grupos o movimientos confieren al cura una espiritualidad vigorosa que le sirve para vivir y afrontar muchos problemas. Hay que preguntarse si todos los aspectos de esa espiritualidad están en sintonía con la condición, la vocación y la misión del cura (...). De una teología específica del ministerio brota una espiritualidad también especifica, estimuladora y exigente... la espiritualidad propia del presbitero, la que surge de las fuentes de la teología del presbiterado, es capaz de conducir al presbítero a una verdadera radicalidad evangélica ${ }^{18}$.

El proyecto educativo del seminario, y, más en concreto, la formación espiritual depende por tanto de esa identidad teológica, de cómo se entienda y se quiere que sea el presbítero que se quiere formar.

\subsection{La pastoreitas como núcleo identificador}

Esta identidad se comprende cada vez más a partir de la imagen bíblica del pastor, a partir de la pastoreitas, mejor que desde las figuras clásicas del rey (la regalitas), del profeta-doctor (magisterialitas), e incluso que desde la del sacerdote (sacerdotalitas), si bien con la conciencia de que la significación de estas figuras, en especial la del sacerdote, se halla incluida en dicha imagen ${ }^{19}$.

18 J. M. Uriarte, Ministerio presbiteral y espiritualidad, Instituto de Teología y pastoral, San Sebastián, 1999.pp.17.19. Todo el Manual antes citado de S. Gamarra se halla en esta perspectiva.

19 Como ejemplo de esta perspectiva en la reflexión teológica puede verse G. Greshake, Ser sacerdote, Sígueme, Salamanca, 2005, quien en su retractatio de la segunda edición afirma: hoy... reconduciría estas diversas formas de integración a la figura originaria del pastor, p.208. La misma perspectiva 
Esta es la perspectiva fundamental y predominante en la misma Pastores dabo vobis ${ }^{20}$, como se advierte con la simple consideración de su título, avalado por la estructura literaria, que viene marcada por una inclusión formada por la frase pastores os daré según mi corazón, tomada del profeta Jeremías $(3,15)$ (cfr. PDV 1 y 82 ). Esta identidad es luego afirmada y descrita en términos de sacramentalidad: el presbítero se configura, se conforma, como un sacramento personal de Cristo Cabeza y Pastor: Los presbiteros son, en la iglesia y para la iglesia, una representación sacramental de Jesucristo Cabeza y Pastor (PDV 15). Mediante la consagración sacramental, el sacerdote se configura con Jesucristo en cuanto Cabeza y Pastor de la Iglesia... Gracias a esta consagración obrada por el Espíritu Santo en la efusión sacramental del Orden, la vida espiritual del sacerdote queda caracterizada, plasmada y definida por aquellas actitudes y comportamientos que son propios de Jesucristo, Cabeza y Pastor de la iglesia y que se compendian en su caridad pastoral (PDV 21). En virtud de su consagración los presbiteros son configurados con Jesús Buen Pastor y llamados a imitar y revivir su misma caridad pastoral (n. 22) .La expresión sacramento de Cristo Pastor así como otras muchas equivalentes, como epifanía, transparencia, manifestación, imagen, instrumento, son constantes en la Exhortación (cfr. nn. 15, 21,22,23).

\subsection{Aspectos fundamentales de la pastoreitas, objetivos de la formación espiritual del presbítero}

Por tratarse de una identidad expresada en un símbolo, por sí mismo lleno de connotaciones teológicas, cristológicas, eclesiológicas, antropológicas y existenciales, un proyecto educativo en la dimensión de la espiritualidad tendrá que explicitar los rasgos fundamentales que la caracterizan y, teniendo en cuenta que la educación es siempre un proceso, priorizarlos a tenor de las circunstancias personales del formando, de su edad y de su proceso de maduración vocacional (cfr. PDV 61).

\subsubsection{La PDV, comentando OT 8, subraya algunos valores y exigencias}

en la Conferencia episcopal italiana, en: Seminarios y vocaciones sacerdotales, nn.17-23. (publicada en SEMINARIOS N. 75, (1980) 49-84. Asimismo en el nuevo Plan, 2006, cfr. n. 4, y especialmente el n. 8, que titula el icono evangélico del Buen Pastor, y que, por lo mismo, considera la caridad pastoral como el centro de la espiritualidad presbiteral (cfr. nn.9-10.18.84).

20 Para una visión amplia de esta perspectiva puede verse mi estudio La formación del pastor en y a la luz de Pastores dabo vobis. Una lectura global y unitaria de la Exhortación, en SEMINARIOS N.125-126 (1992) 333-358. 
fundamentales del camino espiritual del candidato al sacerdocio (n.46), entre los que considera fundamentales los siguientes:

- vivir íntimamente unidos a Jesucristo, con una relación de "verdadera amistad".

- la búsqueda de Jesús y el encuentro con El, para lo cual indica y describe varios medios: lectura meditada y orante de la palabra de Dios (n.47), el silencio; la eucaristía, los sacramentos y la Liturgia de las horas (n 48).

- Buscar a Cristo en los hombres, especialmente en los pobres, y convertirse, por tanto, en el hombre de la caridad (n. 49), que antes ha definido como la caridad pastoral (cfr. n. 23), calificada, como vimos, como el centro unificador de la vida espiritual, y en la que se contempla la radicalidad evangélica, $y$, más en concreto, la obediencia, la castidad celibataria y la pobreza (n. 49,50 , y 28-30).

2.3.2. En el Proyecto elaborado en función de nuestros viandantes se contemplan varias líneas de necesidades formativas que convergen en la dimensión espiritual, y que en general coinciden con las que señalan buena parte de los análisis socio-pastorales de los seminaristas actuales 21 . Estas necesidades especifican los objetivos de formación espiritual, contemplando, por una parte, la necesidad de des-programación o, como ellos dicen ya habitualmente, de des-formatación, de conversión y cambio de mentalidad, de actitud y de conducta, y, por otra, en perspectiva positiva, las metas a conseguir, la adquisición de los valores de la pastoreitas recogidos en Pastores dabo vobis y en el Plan de formación español.

2.3.2.1. La primera, y posiblemente la más frecuente y grave, es el subjetivismo en la comprensión y vivencia de la vocación presbiteral. Se encuentra explícitamente formulada en PDV: No falta la tendencia a concebir la relación con Dios de un modo individualista e intimista, como si la llamada de Dios llegase a cada persona por vía directa sin mediación comunitaria alguna y tuviese como meta una ventaja o la salvación misma de cada uno de los llamados (PDV 37).

Para responder a esta mentalidad el Proyecto educativo deberá fijar como objetivo el reconocer y aceptar con gozo la dimensión ecle-

21 Puede verse L. Trujillo, ¿Los nuevos seminaristas?, SEMINARIOS, n. 167 (2003) 11-28. 
sial inscrita originariamente en toda vocación cristiana y en particular en la vocación presbiteral (PDV 37) o, como se formula aún más claramente en el n. 35: la esencial dimensión eclesial de la vocación cristiana y, en particular, de la vocación presbiteral, que es una llamada, a través del sacramento del orden recibido en la Iglesia, a ponerse al servicio del pueblo de Dios con una peculiar pertenencia y configuración con Jesucristo...Cabeza y Pastor de la Iglesia, y como se explica a continuación, todo presbítero recibe del Señor la vocación a través de la Iglesia como un don gratuito, una "gratia gratis data" (carisma)... El candidato al presbiterado debe recibir la vocación sin imponer sus propias condiciones personales".

2.3.2.2. La segunda, el individualismo, que conoce muchas manifestaciones, como la afirmación del gusto como motivación vocacional y de buena parte de sus decisiones; la organización de la vida desde los propios intereses, visiones y proyectos; la afirmación, en la práctica, de la libertad en términos de autonomía absoluta pretendiendo que sea la única e inexplorable fuente de opciones personales y considerándola a toda costa como afirmación de sí mismo (PDV 37).

A esta línea habrá que responder con el descubrimiento y la aceptación de la vocación como libre diálogo de amor(Ibíd.); con la conciencia de formar parte de una realidad que se define por la comunión y del compromiso de contribuir con su ser y su actividad a realizar la comunión eclesial (PDV 16.22); con la asimilación y ejercicio de la caridad pastoral que pide que, para no correr en vano, trabajen siempre los presbíteros en vínculo de comunión con los Obispos y con los otros hermanos en el sacerdocio (PO, 14) (PDV 23); con la comprensión y acogida cordial de la obediencia apostólica o pastoral, que ha de vivirse en un clima de constante disponibilidad a dejarse absorber, y casi "devorar", por las necesidades y exigencias de la grey (PDV 28).

2.3.2.3. La tercera podríamos calificarla, con un cierto tono caricaturesco, como el solemnismo o gloriosismo. Se manifiesta en el deseo de figurar, con una no disimulada complacencia, en las grandes y solemnes celebraciones; en una cierta mentalidad competitiva ya en los propios estudios y resultados académicos; en una frecuente referencia a los cargos eclesiásticos, no exenta al parecer de aspiraciones a cargos y títulos de la carrera eclesiástica, con una cierta dosis de clericalismo, en vertiente de sacralidad y de ejercicio de poder; en una tendencia no excesivamente controlada a una vida cómoda o sin excesivas privaciones especialmente en lo 
que se refiere a medios técnicos.

La tarea educativa espiritual tendrá que esforzarse por inculcar y asimilar la conciencia de la ministerialidad, de la comprensión del presbiterado como sacramento del Cristo Siervo, que lleva su servicio a su plenitud con la muerte en cruz, o sea, con el don total de sí mismo, en la humildad y el amor, que asume que el que es cabeza del pueblo debe, antes que nada, darse cuenta de que es servidor de muchos... porque el Señor de los señores no se desdeñó de hacerse nuestro siervo (San Agustín); que asimila esa característica de la vida espiritual de los ministros del NT, la actitud esencial de servicio al pueblo de Dios (cfr. Mt 20,24s; Mc 10, 43-44), ajena a toda presunción y a todo deseo de "tiranizar" la grey confiada (cfr. 1 Pe 5,2-3) (PDV 21); que ha de comprometerse en la ordenación a conformar su vida con el misterio de la cruz del Señor( PDV 24).

También habrá que ayudar a descubrir y estimular el sentido de la pobreza pastoral, adquiriendo, en primer lugar, una profunda y cordial disponibilidad a ser enviado allí donde su trabajo sea más útil y urgente, aunque comporte sacrificio; en segundo lugar, el compromiso de no someter el servicio del evangelio y de la Iglesia a las ventajas e intereses que del mismo puedan derivarse; asimismo la capacidad de testimoniar la pobreza con una vida simple y austera, habituados ya a renunciar generosamente a las cosas superfluas (PDV 30, citando OT 9), y, finalmente, prepararse para optar gozosamente por los pobres, por estar al lado de los más débiles,... (de) los pequeños, los pecadores, los marginados de cualquier clase, según el modelo ofrecido por Jesús en su ministerio profético y sacerdotal (PDV 30).

2.3.2.4. Encontramos también un cierto territorialismo eclesiástico, una identificación con el propio terruño de la iglesia particular, con la propia tribu eclesiástica, idealizando la iglesia propia, su clero, su idiosincrasia, sus proyectos pastorales, sus manifestaciones religiosas. La terapia exigirá aquí una abertura no solo a la comunión sino también a la catolicidad, a la misionariedad del ministerio presbiteral diocesano, pues la vida espiritual de los presbiteros debe estar profundamente marcada por el anhelo y el dinamismo misionero (PDV 32).

2.3.2.5. Finalmente encontramos también dos aspectos muy bien señalados y analizados por Mons. Uriarte 22 , el de la exterioridad, la

22 Cfr. Ministerio presbiteral y espiritualidad, pp. 28-30. 
superficialidad, así como una marcada tendencia a los mínimos, a la ley del esfuerzo no más del necesario. El primero acentuado hoy por la facilidad de contactos, de mensajes, de mariposeo y casi juego en todos los aspectos, sin hondura en las relaciones. El segundo, especialmente significativo en el esfuerzo en los estudios, con una falta de motivación profunda apostólica y pastoral, con escasa profundización en los temas, con la facilidad para el uso de medios técnicos que ahorran el esfuerzo de la asimilación. Tendencia que se combatirá con la consideración del radicalismo evangélico exigido a cualquier discípulo y especialmente motivado en el presbítero por su sacramentalidad y por su responsabilidad ministerial, una de cuyas manifestaciones fundamentales será la entrega amorosa total, exclusiva y plena, esponsal, que comporta la castidad celibataria (cfr. PDV 23.25.29.49-50).

\section{MEDIOS Y ACTIVIDADES PARA LA FORMACIÓN ESPIRITUAL}

El proyecto educativo, además de la claridad en señalar el objetivo general y las especificaciones del mismo, que definen los diferentes aspectos de la identidad del presbítero, debe contemplar e incluir los medios y actividades, -los instrumentos dirá la PDV (n.61)- más adecuados a la consecución de los mismos así como algunos ritmos temporales precisos (PDV 61).

Por lo que se refiere a la formación espiritual, enumera, describe, aconseja y en ocasiones urge algunos, todos ellos recogidos y concretados después tanto por los numerosos tratadistas de la formación espiritual como en los diferentes Planes nacionales. Así, en cuanto al objetivo de la búsqueda de Dios, encontramos: la lectura meditada y orante de la palabra de Dios y la oración (n.47), la eucaristía diaria y la Liturgia de las Horas, y el sacramento de la Reconciliación (n.48), todos ellos, como se advierte, en el ámbito de la práctica religiosa. En cuanto a la búsqueda de Cristo en los hombres se señala: el encuentro con el prójimo; la entrega generosa y gratuita, -favorecida por la vida comunitaria-; el servicio humilde y desinteresado -para lo que puede servir una justa, profunda y tierna, a la vez, devoción al corazón de Jesús-; la formación de la caridad, en particular del amor preferencial por los pobres. En este contexto de la caridad, se señalan la obediencia, la pobreza y el celibato (n. 49), dedicando un número entero a la descripción e importancia de éste, sugiriendo como medio la solicitud del obispo y la vida fraterna entre los sacerdotes, y que se debe presentar el celibato con claridad, sin ninguna ambigüedad y de forma positiva y que el seminarista conozca la natura- 
leza cristiana y verdaderamente humana y el fin de la sexualidad en el matrimonio y en el celibato (n.50).

En el proyecto educativo que presento se contemplan los dos tipos de medios, teóricos o de orden intelectual, de formación de la mente, y prácticos, o de ejercitación de la voluntad en orden a la asimilación de valores y actitudes que lleven a adquirir hábitos conformadores de la vida concreta, de acuerdo a la pauta que la PDV, como la buena pedagogía, que a propósito de la formación pastoral, indica que se desarrolla mediante la reflexión madura y la aplicación práctica (PDV n. 57).

En cuanto a los ritmos temporales el proyecto sigue las pautas ofrecidas por la Conferencia Episcopal Española en el Plan de formación, en el que el itinerario educativo se estructura en tres etapas.

La primera, que coincide de ordinario con los dos primeros años del seminario mayor o etapa filosófica, tiene como objetivo fundamental la objetivación y verificación de la opción vocacional ya tomada en el momento del ingreso... Los seminaristas... han de pasar de una visión predominantemente subjetiva e idealista a una más objetiva y realista de sus propias motivaciones, actitudes y opciones, así como también de su concepción del ministerio presbiteral al que aspiran (Plan, n.195).

La segunda, la de la teología, de tres o cuatro años, tendrá como objetivo la asimilación e identificación con el ser y el ministerio del presbítero diocesano (n.202), con especial referencia a la consolidación en la opción celibataria y al descubrimiento y vivencia de la fraternidad sacramental del presbiterio y de la acción ministerial solidaria (Ibíd. n. 203).

La tercera, o etapa pastoral, se centra principalmente en la asimilación de la teología y teoría de la acción pastoral y en una experiencia práctica del ministerio previa a la ordenación presbiteral (Ibíd. n. 210 ss).

El programa teórico que presento como parte del proyecto se centra especialmente en la segunda y tercera, aunque contemplando elementos que serían más propios de la primera ${ }^{23}$. El programa se desarrolla com-

23 Los objetivos de la etapa primera, así como los de la introductoria, o propedéutico, se contemplan en parte también aquí pero de manera menos acentuada, porque han sido objeto de otros programas, que contemplan fundamentalmente una adecuada introducción a la vida espiritual, la iniciación a los diversos métodos de oración con ejercicios prácticos, introducción e iniciación a la oración de los salmos y a la Liturgia de las Horas, psicología y antropología de la vocación, comprensión y aceptación del proyecto educativo global del seminario mayor, iniciación a la vida comunitaria, conocimiento 
pleto a lo largo de los varios años, debido al condicionamiento del horario académico y del resto de la organización de la vida comunitaria. Los diferentes temas se desarrollan ampliamente, de ordinario en varias sesiones según lo requiera la materia tratada.

\subsection{Formación teórica: Identidad-espiritualidad del presbítero dioce- sano secular}

3.1.1. El presbiterado como misterio de gracia o la gracia de ser llama dos para el ministerio presbiteral.

- Teología bíblica de las vocaciones individuales;

- Dimensión eclesial de la vocación al presbiterado: el llamamien to de la Iglesia;

- Dimensión antropológica: soy llamado, luego existo;

- Llamados por el propio nombre para el presbiterado (PDV 3537).

3.1.2. Las claves de la comprensión teológica actual del ministerio presbiteral: visión general: la sacramentalidad, la ministerialidad, la relacionalidad, el carisma de totalidad (PDV 15-16).

3.1.3. La ministerialidad: sacramentos del Cristo Siervo.

- Cristo Siervo: sentido bíblico, teológico, eclesiológico y antropo lógico (Ictura y comentario de los textos bíblicos de Isaías y del NT sobre el Siervo de Yavé).

- El presbiterado como ministerio: la autoridad-servicio, la renuncia al señorío", a los medios ricos y poderosos, la pobreza pastoral y la opción por los pobres (PDV 13-15.21.30).

3.1.4. Sacramentalidad: sacramentos de Cristo Pastor: La pastoreitas como núcleo identificador del ministerio presbiteral. (Textos bíbli cos y PDV 13-15).

- La imagen y figura del Pastor en la Biblia (A y NT)

- Funciones del pastoreo en la Escritura

- Aplicación al ministerio apostólico y a los presbíteros

3.1.5. La caridad pastoral, como centro especificador y unificador de la espiritualidad propia y del presbítero: contenidos, manifestaciones y realizaciones (PDV 14.21.22.23).

y experiencia de la riqueza y trascendencia de las relaciones interpersonales, cultivo de la sensibilidad ante las situaciones de miseria de la condición humana, especialmente aptas en orden a la formación del corazón pastoral, de la caridad pastoral 
3.1.6. El radicalismo evangélico: en general (PDV 27).

3.1.6.1. La pobreza pastoral(PDV 30)

3.1.6.2. La obediencia pastoral (clave de la relacionalidad):

- Colaborador del Obispo (LG 28; PO 7; PDV 16.17.28.74)

- La fraternidad presbiteral; vocación para la comunitariedad;

- La amistad y las amistades;

- Relaciones con los presbíteros religiosos;

- Relaciones con los laicos;

3.1.6.3. La ministerialidad de los laicos.

3.1.7. El celibato pastoral (PDV 29.44.50)

- Lo que significa y comporta

- Los motivos de la opción celibataria

- La formación para la castidad celibataria

- Cuestiones especiales: masturbación, homosexualidad, pedofilia...

3.1.8. El carisma de totalidad:

- La diocesaneidad (PDV 31.68.74)

- La secularidad, sus manifestaciones y exigencias

- La espiritualidad en el ejercicio del ministerio: las funciones

- El modo de hacer: la forma y la figura: de funcionario

a vocacionado

- La oración pastoral: liturgia de las Horas, la intercesión, la bendición; la oración personal

- La Lectio divina individual y en grupo

- El ministerio de la reconciliación

- El acompañamiento espiritual individual

- La nueva evangelización: sentido, contenidos, métodos.

\subsection{Formación práctica: Medios y actividades}

Más importante que la información o enseñanza teórica es la formación mediante la aplicación práctica. En el proyecto educativo que presento, se contemplan varios medios prácticos, que tienen que ver con los que tradicional y habitualmente se señalan como los medios más adecuados, en este caso orientados y condicionados a partir del objetivo general señalado más arriba.

\subsubsection{La comunidad educativa.}

El primero lo constituye, sin duda, la comunidad educativa, el ambiente educativo del seminario, el estilo de vida y la organización misma del seminario, su funcionamiento interno. 
La PDV subraya la prioridad de este medio cuando, entre los ambientes propios de la formación sacerdotal, sitúa en el primer lugar a la comunidad formativa del seminario mayor (cap. V. II, n. 60-62), y, al exponer los agentes protagonistas de la formación sacerdotal, describe con amplitud y profundidad la función de la comunidad inmediatamente después de las de la Iglesia y el Obispo (V, III, nn. 65.66).

Se comprende esta prioridad cuando se entiende que se trata de la comunidad promovida por el Obispo para ofrecer... la posibilidad de revivir la experiencia formativa que el Señor dedicó a los Doce, o de ser, a su manera, una continuación en la Iglesia de la íntima comunidad apostólica formada en torno a Jesús, en la escucha de su palabra, en camino hacia la experiencia de la Pascua, a la espera del don del Espíritu para la misión (PDV 60), con una específica contribución al crecimiento pastoral y apostólico de los alumnos. Los diversos miembros de la comunidad del seminario... colaboran... al crecimiento de todos en la fe y en la caridad para que se preparen adecuadamente al sacerdocio, (PDV 60d); de una comunidad eclesial, como comunidad de discípulos del Señor en que se celebra una misma liturgia (que impregna la vida del espíritu de oración), formada cada día en la lectura y meditación de la palabra de Dios y con el sacramento de la eucaristía, en el ejercicio de la caridad fraterna y de la justicia (PDV 60 e).

Por eso no se olvida de indicar que el seminario, antes que ser un lugar o espacio material debe ser un ambiente espiritual, un itinerario de vida, una atmósfera que favorezca y asegure un proceso formativo de manera que el que ha sido llamado por Dios al sacerdocio pueda llegar a ser, con el sacramento del orden, una imagen viva de Jesucristo Cabeza y Pastor de la Iglesia (PDV 42), y que es esencial para la formación de los candidatos al sacerdocio y al ministerio pastoral-eclesial por su naturaleza- que se viva en el seminario no de un modo extrínseco y superficial, como si fuera un simple lugar de habitación y de estudio, sino de un modo interior y profundo: como una comunidad especificamente eclesial, una comunidad que revive la experiencia del grupo de Los Doce unidos a Jesús (PDV 60).

Esto significa que se considera como factor formativo de primera magnitud, incluso en la dimensión espiritual, la estructura misma del seminario, la organización de la vida, el lugar y momento y estilo de las celebraciones, especialmente de la Eucaristía y de la Liturgia de las Horas, el tipo de piedad y, por lo mismo, de prácticas en que se expresa, el clima de silencio que se establece, la dispersión de vidas y actividades con su automática orientación hacia la exterioridad o la fragmentación. Y, sobre todo, el tipo de relación que se establece, tanto de los educadores entre ellos y con los seminaristas como de éstos entre sí, así como la concien- 
cia de los propios seminaristas sobre su responsabilidad y participación en la formación y discernimiento de la propia vocación y aun la de sus compañeros, en cuanto miembros de una auténtica comunidad eclesial diocesana. Todo ello infunde el estilo de presbítero, de pastor que se pretende formar, y va configurando, como por ósmosis, por contagio, el modelo o paradigma de iglesia y del presbítero en ella, que ellos querrán encarnar.

Esto se concreta, en primer lugar, en la conformación y configuración del equipo educativo, en nuestro caso, un equipo mixto en edades $(40,50$ y 60 años en sus comienzos, ahora 50,60 y 70), en procedencias (un portugués y dos españoles), en sensibilidades (un presbítero diocesano focolar y dos operarios), en buena sintonía entre ellos aunque con talantes diferentes, con entusiasmada vocación educadora de presbíteros, en comunión plena en cuanto al tipo de presbítero a formar.

$Y$ con unas convicciones comunes en cuanto a la labor educativa: la de formar un auténtico equipo de vida y de trabajo (cfr. Directrices); la de saberse todos corresponsables de la formación en todas las dimensiones, aunque desde responsabilidades o funciones diferentes; en vivir internos todos formando in quantum humana fragilitas una familia (OT 5), una única comunidad con los seminaristas que se traduce en participar con ellos habitualmente en los actos comunitarios, especialmente los litúrgicos; en formar parte de los equipos de trabajos comunitarios (como el de comedor y cocina); en la dedicación cuasi-exclusiva, -disponibles las 24 horas del día- a la atención de los seminaristas, sin comprometerse con otras funciones o cargos que sean obstáculo para esa dedicación plena.

Este medio ha contribuido eficazmente a crear una conciencia y sensibilidad de eclesialidad y misionariedad, de comunión y fraternidad presbiteral lo que ha creado actitudes y anhelos de vida y trabajo en equipo y hasta de vida común (cfr. PDV 66), de valoración de la actividad orante en el ministerio de los presbíteros.

Se concreta también, en segundo lugar, en la incorporación de los seminaristas como protagonistas de su propia formación (cfr. PDV 69). Esto se realiza participando ellos todos los cursos, una vez formulado el objetivo general por parte del equipo educativo (la meta absoluta claramente definida, PDV 61), en la programación del curso, determinando los objetivos específicos y concretando las actividades y medios comunitarios adecuados; realizando al terminar el primer semestre, una evaluación comunitaria, según la cual se reafirman o corrigen o se añaden objetivos o medios; evaluación que vuelve a realizarse al terminar el curso, que contribuye a marcar los objetivos para el año siguiente. Esta formulación 
de objetivos y medios se hace todos los años en conexión con los acontecimientos y los proyectos pastorales de la iglesia universal y de las respectivas iglesias diocesanas. Con ello creemos se afianza la conciencia eclesial, la necesidad de que el presbítero cuente con la comunidad respectiva en la formulación, realización y evaluación de los proyectos parroquiales.

En tercer lugar, en la responsabilización de varios de los servicios -ministerios comunitarios, podríamos llamarlos- que la vida comunitaria supone y exige, como son los de la oración comunitaria y litúrgica, en este caso, con el asesoramiento y orientación del director espiritual o del formador encargado de presidir las celebraciones, en la que se alternan semanalmente todos los miembros del equipo; los del servicio del comedor todos los días y de cocina, los sábados y domingos; y varios otros de menor entidad pero no menos formativos (como sacristanes, acólitos, maestro de ceremonias para las celebraciones en la catedral, cantores, biblioteca, bar, animación misionera, hoja informativa Seminarios, que se publica trimestralmente, deportes, animación festiva, servicio de internet). No es poca la ayuda que este medio ofrece en orden a crear actitud de servidores, de servicios gratuitos, escondidos, humildes, cosa que se afianza con la evaluación comunitaria correspondiente en los tiempos arriba señalados.

En cuarto lugar, se ha concedido una gran importancia a las relaciones humanas, tanto en su vertiente de conocimiento de los mecanismos de las mismas, como en la vertiente práctica de establecimiento de unas relaciones interpersonales profundas, fraternas, no solo como factor de socialización, sino como experiencia de fraternidad eclesial. Esto es especialmente necesario en una comunidad como la nuestra, -interracial, intereclesial, e internacional-, y como preparación para comprender y anticipar la vivencia de la fraternidad presbiteral. Para lo cual se han programado cada tres o cuatro años, cursillos específicos tipo taller, teóricoprácticos, de relaciones humanas, lo que ha facilitado la integración de los grupos, ha posibilitado un clima de comunicación habitual de buen nivel entre todos, y ha ayudado a superar en buena parte la dificultad que supone el compartir la palabra, la comunicación de fe, la narración de la propia vocación, y aun de la experiencia de Dios. De esta forma se ha hecho posible inicialmente implicar a los alumnos en la evaluación vocacional de los compañeros con ocasión de la institución en los ministerios y en los procesos de ordenación.

\subsubsection{Las "formas de expresión"}

Tomo esta formulación de Mons. Uriarte cuando hablando de la espiritualidad sacerdotal sugiere la necesidad de encontrar nuevas formas de 
expresión ${ }^{24}$.

Es verdad que en los seminarios han caído buena parte de las expresiones o prácticas que jalonaban, como ya indiqué, los días, los meses, y el año, que se consideraban como manifestaciones de una vida espiritual floreciente. Han caído porque se comprende que no son manifestaciones ni mediaciones adecuadas en la nueva comprensión de la espiritualidad y de la presbiteral.

Estas son, repartidas en los diferentes ritmos temporales, las formas de expresión incorporadas en este proyecto educativo.

3.2.2.1. En el ritmo diario, la oración de la mañana, que de ordinario consiste en las Laudes de la Liturgia de las Horas, pero que a veces se sustituye por otros tipos de oración, en orden a seguir conociendo y experimentando diferentes y variados métodos de oración. Al terminar la oración se lee el evangelio del día, como preparación remota para la eucaristía.

La celebración de la Eucaristía tiene lugar al final de la jornada, al caer de la tarde, antes de la cena, resaltando así el carácter de culmen del día, al vernos impedidos de situarla en el centro del día por el condicionante académico. En la Eucaristía se integran habitualmente las Vísperas.

En la oración de la noche se alternan varias expresiones: los lunes se reúnen por diócesis, con el fin de que todos puedan alimentar y mantener el fuego vivo de la diocesaneidad; los martes y viernes, se rezan comunitariamente las Completas, incorporando a veces diferentes modelos de evaluación del día o examen, especialmente el ignaciano; otros dos días, miércoles y sábados, dada la especial sensibilidad mariana que existe en Portugal por influencia del fenómeno llamado Fátima, se ha incorporado el rezo del rosario en comunidad. De esta manera se responde a las diversas sensibilidades existentes en la comunidad, y se va adquiriendo un respeto por la diversidad de expresiones así como un cultivo de las mismas, necesario para su futuro ministerio pastoral.

3.2.2.2. Semanalmente se ha incorporado el jueves la hora santa de adoración al Santísimo, siguiendo con frecuencia la orientación de alabanza en la línea de Taizé, -a la que los jóvenes se muestran especialmente sensibles-, y con la oración por las vocaciones. Los domingos, debido a los horarios de las actividades apostólicas, la comunidad solo se reúne para las Vísperas al caer de la tarde, y para las Completas por la noche.

24 Cfr. Ministerio presbiteral y espiritualidad, Publicaciones Idatz, San Sebastián 1999, p.26. 
Desde hace ya varios años se ha incorporado, después de un período de formación teórica, la práctica de la lectio divina en grupo. Esto se hace los viernes, antes de la eucaristía, con puesta en común durante la celebración de la misma.

3.2.2.3. Como práctica mensual, se ha programado el rezo del rosario todos los días durante los meses de octubre y de mayo, aunque los jueves se mantiene la adoración al Santísimo.

3.2.2.4. En el ritmo anual, la forma de expresión sin duda más significativa y de extraordinario aprovechamiento es la de los ejercicios espirituales ignacianos, de ocho días íntegros, en riguroso silencio, y orientados por Padres Jesuitas según el conocido método de S. Ignacio. Se programan para antes de comenzar el curso y para todos los alumnos de la segunda etapa, desde el tercero al sexto año.

Los alumnos de los dos primeros cursos participan en unos ejercicios espirituales de tres o cuatro días, en el primer mes del curso, siguiendo el mismo método, aquí orientado o por los propios PP. Jesuitas, o por alguien que haya completado el curso para directores de ejercicios organizado por ellos. Así se van iniciando ya en el método, pero sin el rigor del mismo, que para ellos, dada su edad y formación, nos parece no resultaría adecuado.

Al comienzo del segundo semestre, y como complemento de la evaluación ya mencionada, se programa una segunda sesión de ejercicios espirituales para toda la comunidad, de tres-cuatro días, fuera del seminario, orientado preferentemente por seglares o religiosas pero también de la escuela ignaciana. Hemos comprobado que este retiro suple con creces los mini-retiros mensuales de una tarde, por lo que estos se mantienen solo en casos excepcionales, con ocasión de los tiempos litúrgicos fuertes.

Los viernes de cuaresma se sustituye el rosario por el Via Crucis; y se participa durante toda una noche en la práctica de la adoración al Santísimo conocida como Laus perennis, práctica que se mantiene en todas las diócesis de Portugal, encargándose cada día una de las parroquias de la diócesis. Esta práctica de la Laus perennis es muy apreciada por los alumnos, y la organizan varias veces a lo largo del año, como vigilia de oración, sobre todo en las vísperas de las ordenaciones.

Se mantienen dos Novenas y un Triduo: la novena de la Inmaculada, que se celebra solemnemente en la catedral, y que incluye una celebración penitencial con confesión individual el último día, del que se encarga la comunidad del seminario; y la de $\mathrm{N}^{a} \mathrm{~S}^{a}$ de la Purificación, o de las Candelas, la patrona del Seminario, celebrada en la capilla del seminario, 
y preparada y solemnizada por los propios seminaristas, bajo un tema general que corresponde al proyecto pastoral de la diócesis, lo que ayuda a fomentar la conciencia de pertenencia a la Iglesia particular; el Triduo, en honra de Nuestro Señor de los Pasos, -una imagen del Señor con la cruz- organizado por una Cofradía pero encomendado habitualmente al Seminario, que se celebra el segundo domingo de cuaresma, e incluye una procesión solemne por la ciudad, y contempla algunas de las estaciones (pasos) del Vía crucis.

\subsubsection{Las experiencias fundantes}

Es sabido que las experiencias fundantes, una categoría de la psicología social, son aquellas en las que la persona se siente envuelta desde sus cimientos, afectada en sus entrañas, lo que produce un vuelco en su estar en el mundo, en su comprensión de la realidad y en su situarse ante ella25. En y por ellas se produce una real conversión mental y existencial. Por eso, en el proyecto que describo se han incorporado varias de estas experiencias, algunas orientadas a todos, otras destinadas para algunos alumnos en particular.

\subsubsection{Experiencias fundantes existenciales}

Entre las generales se incluyen las ya descritas en el ritmo anual, la de los ejercicios espirituales ignacianos, en los que el silencio, la soledad, y el método provocan un auténtico revulsivo en los seminaristas marcados, como la generación joven actual, por el ruido, la superficialidad, la dispersión, la exterioridad.

Se incluyen también las conocidas como vacaciones misioneras, un período entre quince y veinte días durante las vacaciones académicas, dedicado a hacer presente la Iglesia en comunidades rurales, tierras de auténtica misión, en contacto con realidades descristianizadas, por una parte, y de marginación social, por otra. Esta experiencia obliga a una fuerte convivencia, lo que confirma y acrecienta la conciencia de fraternidad, pero además, y sobre todo, los enfrenta con situaciones de indiferencia, de hostilidad y aun de rechazo, lo que purifica las motivaciones

25 Para la fundamentación de este apartado puede verse, A. Tornos, La vida cotidiana, campo de evangelización, SAL TERRAE 81(1993)437-451; a las experiencias fundantes y de choque en el campo de la evangelización alude F. Sebastián Aguilar, Nueva evangelización. Fe, cultura y política en la España de hoy, Encuentro, Madrid 1991, p.158. 
sacrales, gloriosas, y los hace realistas frente al ministerio deseado, los sensibiliza para el ministerio de la intercesión, les descubre las dificultades que van a encontrar en su futuro ministerio, y al comprobar su propia incapacidad y la necesidad de recursos múltiples para la acción apostólica, los motiva para una mayor y mejor preparación intelectual y metodológica.

De choque se puede calificar también la visita al Cenobio de la Cartuja, visita guiada por el Prior de la misma, que les pone en contacto con la sobriedad y pobreza de las celdas, con el silencio y la soledad, con la contemplación y la vida entregada a la oración, de la que salen profundamente impresionados.

En el itinerario educativo se contempla también otra experiencia, -esta bien puede ser calificada como de choque- aunque esta tiene lugar en el período propedéutico. Se trata de un servicio de voluntariado durante quince o veinte días en un hospital psiquiátrico de deficientes profundos y muchos terminales, bajo el acompañamiento de los $\mathrm{HH}$. de $\mathrm{S}$. Juan de Dios. Ahí descubren un mundo de miseria absoluta, de deshechos humanos, que les provoca repugnancia hasta impedirles comer en los primeros días, pero despierta la sensibilidad y los prepara para el ministerio con los marginales y los marginados de la sociedad.

Aunque menos de choque, esta experiencia se continúa posteriormente en las etapas del ciclo institucional, promoviendo, en los primeros años, el acompañamiento lúdico-celebrativo con deficientes, en residencias de la tercera edad, y, en los años de teología, la visita a los enfermos en el Hospital de la ciudad, orientados por el capellán, y ya dentro de las experiencias pastorales. En algunos años se han añadido las visitas a la cárcel de la ciudad, aunque por la situación y degradación de ésta se nos ha desaconsejado por parte de los responsables pastorales de la misma.

\subsubsection{Experiencias fundantes celebrativas}

En el ámbito del seminario, nos resulta especialmente significativa la que se denomina misa internacional, animada en sus diferentes partes por cada uno de los grupos diocesanos que conforman la comunidad Algarve, Évora, antes, Timor y Angola, y ahora Cabo Verde y Ucrania-. Aprender y cantar todos los alumnos los cánticos respectivos de las diferentes iglesias y en diferentes lenguas provoca una experiencia emocional de la catolicidad con una hermosa proyección sobre las relaciones interpersonales en la rutina de la cotidianidad.

Se contempla también en el proyecto la participación en todas las celebraciones de la Catedral, bastante frecuentes en la Archidiócesis, presididas por el Sr. Arzobispo, que hacen posible, por una parte, la experien- 
cia del esplendor celebrativo, extraordinariamente cuidado en esta Iglesia, el encuentro con el presbiterio diocesano representado por el clero de la ciudad, y en varias ocasiones por el del resto de la diócesis, como en el Triduo sacro, y con el numeroso pueblo de Dios que participa en estas celebraciones catedralicias, con una profunda conciencia de iglesia diocesana.

Asimismo se contempla también la participación del seminario en las celebraciones penitenciales organizadas tanto en el nivel de la ciudad entera, durante los tiempos litúrgicos fuertes, como en las que se celebran en las diferentes parroquias donde prestan su apoyo pastoral.

Finalmente se incluye, también en ocasiones especiales, la participación como peregrinos en las solemnes celebraciones del Santuario de Fátima, que siempre causan en todos un profundo impacto socio-religioso, de comunión con la fe del pueblo sencillo, de devoción, confianza y entrega mariana, de sana y positiva crítica de prácticas y expresiones devocionales o sacrificiales exageradas.

\subsubsection{La atención al viandante (PDV 61)}

El fin específico del seminario que es el acompañamiento vocacional de los futuros sacerdotes, $y$, por tanto, el discernimiento de la vocación, la ayuda para corresponder a ella y la preparación para recibir el sacramento del orden no puede limitarse a un proyecto educativo comunitario, a pesar de la importancia que le hemos concedido, por muy claramente que esté definido y por adecuados que sean los medios usados. Exige también la atención a cada persona, el viandante, en su concreta y personal circunstancia, lo que se realiza a través de la dirección espiritual. Y una dirección personalizada, ya que las distintas formas comunitarias de dirección espiritual, de intercambio de experiencias y de revisión de vida, no deben jamás sustituirla26.

La dirección espiritual está encomendada al director espiritual oficial, pero, de acuerdo con las citadas Directrices (n. 44), hay otros dos sacerdotes de la diócesis designados por el Sr. Arzobispo, como garantía de la libertad de elección por parte del seminarista.

El director espiritual oficial forma parte de la comunidad educativa, forma equipo con los otros formadores. Tiene dedicación cuasi-exclusiva, y, en el caso que nos ocupa, ejerce con frecuencia también una función de tutoría u orientador de estudios. Comparte con los alumnos la vida, la ora-

26 Directrices para la formación de los formadores, n.61. 
ción, las comidas. Participa en los servicios comunitarios junto con los alumnos, con positiva sorpresa e influencia en este sentido por parte de ellos, sobre todo los que vienen de otras iglesias y modelos formativos, de ordinario acostumbrados a ver y respetar la distancia, sacralidadsuperioridad de la figura. Preside la eucaristía comunitaria la semana que le toca, con lo que, mediante las homilías, ofrece doctrina, criterios, orientaciones, no solo de orden religioso, sino sobre como espiritualizar la vida ordinaria, sobre todo en el ámbito de las relaciones y en el de la formación intelectual. Con todo ello se transmite el mensaje de que la dirección espiritual no es algo ajeno, exterior, yuxtapuesto, superpuesto o contrapuesto al proyecto educativo del seminario, y menos a los otros educadores.

La función fundamental es ayudar al muchacho a hacer una lectura de su vida, a eso que san Agustín llama la amans memoria Dei, el descubrimiento de la voluntad de Dios sobre el joven y por tanto el discernimiento vocacional, descubriendo los puntos altos de la experiencia de Dios, uniéndolos entre sí como formando un mapa que ayuda a descubrir la orientación profunda, el sentido hondo de la vida, encaminada suavemente, atraída profundamente por un agente que domina, conoce y conduce los acontecimientos. Esta función se completa y perfecciona con la iluminación sobre los valores teológicos, cristológicos, eclesiológicos y antropológicos de la vocación y del ministerio presbiteral, sobre la valoración y acogida de las mediaciones eclesiales como presencia y manifestación de Dios; es también paño de lágrimas en los momentos de duda, dificultad, ayudando a superar miedos y obstáculos, a asumir y superar las propias incoherencias en la vida de cada día ${ }^{27}$.

27 Véase la descripción hermosa de esta función en A. Mattheuws, La direction spirituelle, Vie consacrée 69(1997)8-28, pp. 12-14. Todo camino de acompañamiento es un retorno al origen del ser personal. Pero este retorno no es recuerdo nostálgico, simple declinación en la identidad, análisis psicológicos, relato descriptivo. Se trata, tanto en los primeros momentos de la relación personal como en los posteriores, de hacer memoria y de guardar en la memoria viva los dones recibidos como el rastro del don de aquel que se confía a nosotros. Para todo hombre reencontrar su origen es tomar conciencia con fuerza y paz del acto creador que lo ha puesto en el mundo y que continúa sosteniendo su existencia a cada instante... Este retorno al origen es el "fundamento" de todo camino espiritual.

El hombre es entregado a sí mismo: descansa en su origen si hace memoria del don y de los dones recibidos, lo que él es. Si el director espiritual invita a su dirigido a hacer memoria de su vida, a releer su vida, es para fortalecer su identidad de criatura y vivificar la certeza de la providencia de los dones de 
El servicio se desarrolla mediante la entrevista personal, tal como ha sido explicada y estructurada por los expertos en el acompañamiento terapéutico, aunque sin el profesionalismo y la rigidez que en ellos se observa ${ }^{28}$. En el director espiritual prevalece la paternidad sobre la profesionalidad.

Dios. Esta toma de conciencia de la historia santa de cada uno es una condición necesaria para comprender el momento actual de su vida. El sentido espiritual de los sucesos no brota únicamente del instante presente. Se lee en la memoria de una historia personal. Es necesario "visitar" y "revisitar" su historia para descubrir en ella el rastro de Dios, el sentido de los acontecimientos. Es la base sobre la que ambos pueden edificar el momento presente, diseñar el futuro: opciones y mociones diversas aparecen siempre en el horizonte de una memoria santa. Este "espesor" del pasado no es pura tiniebla, aun cuando el acto de memoria puede revelar sufrimientos y agujeros negros. El acto de memoria surge de la libertad: esta permite a cada uno asumir sus debilidades conociéndolas lucidamente. Ella permite así a la gracia de Dios ser "perdón, resurrección y vida transfigurada". Hacer memoria es enraizarse en la certeza de que los agujeros negros y los sufrimientos de la historia personal han sido asumidos por Dios. Dios ha estado presente en cada instante.

El director espiritual desarrolla la certeza de esta presencia y llama al dirigido a vivir de ella cada vez más profundamente. Hacer memoria es poner un acto de confianza, es renovar su confianza. El sujeto puede releer varias veces su vida. Este ejercicio realizado fraternalmente a veces ante personas diferentes, debe conducirlo a crecer en la comunión con Dios, más íntimo a él que él mismo. El descubrimiento espiritual de cada uno pasa por esta memoria. Ella se enriquece con lecturas diversas. El ser humano descubre ahí cada vez más su verdadera identidad de hijo de Dios.

El acto de memoria es un acto de consuelo. Da frutos de paz y de gozo, aun cuando el hermano haga memoria de sufrimientos y de errores. Los sufrimientos, como el pecado, forman parte de la vida del hombre. Dios da al ser humano el poder curar sus sufrimientos y recibir el perdón. Haciendo memoria libremente el hombre se dispone a esta acción divina. A veces es necesario un largo camino espiritual para poder encontrar la paz y el gozo y recibir la certeza de estar "en las manos de Dios", de saborear la ternura de un Padre que nos ama desde toda la eternidad, de unirse íntimamente al grito de los pecadores perdonados y gritar en verdad el grito de la liturgia pascual: 'feliz culpa que nos ha merecido tan grande redentor ( pp.12-14).

28 Una exposición clara y adaptada a la dirección espiritual de esta técnica puede verse en B. Giordani, Una nueva metodología para la dirección espiritual, SEMINARIOS 28(1982)147-162. 
Las entrevistas son marcadas por los mismos muchachos, para garantizar mejor su libertad y que se realicen cuando ellos lo necesitan. La plena disponibilidad del director y su presencia permanente en el seminario hace viable esta atención en el momento oportuno.

Como instrumento se ha explicado y favorecido la elaboración y el seguimiento del proyecto personal de vida. Éste contempla, en primer lugar, el análisis de la propia realidad y situación en cada una de las dimensiones por parte del muchacho, ayudado por el director espiritual. Este análisis lleva a establecer un diagnóstico de la realidad personal en cada una de esas dimensiones, lo que se traduce con facilidad en concretar los respectivos objetivos, así como los medios o actividades adecuadas para la consecución de los mismos, con los lugares y tiempos de realización de esas actividades. La labor del acompañante en todo el proceso es completar, matizar, sugerir, confirmar y, en sucesivas entrevistas, evaluar tanto los objetivos como el cumplimiento de los compromisos, con la consiguiente reorientación en caso de necesidad, o la determinación de nuevos objetivos una vez comprobada la suficiente consecución de los anteriores 29 .

Dentro de este proyecto personal ocupa un lugar de primer orden el tiempo dedicado a la oración individual y a la lectura espiritual, situado por cada uno en el momento del día para él más adecuado. En él se incluyen también, aunque sin tiempos específicos lo que hemos venido en llamar las visitas de amistosa cortesía al Santísimo al pasar por delante de las capillas u oratorios de la casa.

El punto más delicado de la actuación es sin duda el descubrimiento y la toma de conciencia por parte del alumno de las motivaciones vocacionales, asunto tan complejo como la psicología y la espiritualidad clásica nos han ayudado a descubrir. En ocasiones habrá que recurrir a personal técnico que con sus medios apropiados puedan ayudar a detectar los condicionamientos inconscientes o subconscientes tanto de la personalidad como de la decisión vocacional.

Al final, después de todos los proyectos, de todos los planes, de todos los esfuerzos, de todos los sufrimientos, tenemos que exclamar: siervos inútiles somos, hemos hecho lo que nos parecía que debíamos hacer (cfr. Lc 17,10$)$ y de la mejor manera que supimos. Pero convencidos de que

29 Para esto se ha elaborado, a partir de un trabajo realizado por D. Julio García Velasco en el Instituto Vocacional Maestro Ávila, una Guía para las conversaciones con el Director espiritual, que ofrecemos en Anexo. 
el agente protagonista primero y último de la formación es el propio Espíritu del Señor, de que El viene en ayuda de nuestra flaqueza, él conoce lo que hay en el hombre, penetra y discierne los corazones, los ilumina, los calienta, los dinamiza, mueve y dirige a la meta deseada, no cesamos de interceder por nuestros jóvenes con constantes gemidos inefables. Y, en nuestra despedida para la ordenación, nuestro último servicio es decirles, desde el corazón, como san Pablo a los presbíteros de Efeso, Ahora os encomiendo a Dios y a su Palabra de gracia, que tiene fuerza para que crezcáis en la fe y para haceros partícipes de la herencia reservada a los consagrados (Hech 20,32).

ANEXO

GUIA PARA LAS ENTREVISTAS CON EL DIRECTOR ESPIRITUAL OBJETIVOS DE LA FORMACIÓN VOCACIONAL

1. DIMENSIÓN INDIVIDUAL (realidad personal)

\section{Valores}

- Conocimiento y aceptación de mí mismo.

- Autodominio y responsabilidad: orden personal, organización del día, de la habitación, puntualidad...

- Capacidad y actitud de silencio interior y exterior.

- Equilibrio emocional-afectivo: reacciones, actitud ante el dolor,

el sufrimiento, los fracasos, las amistades, la sexualidad-castidad.

- Austeridad, renuncias.

- Voluntad, decisiones, constancia, fidelidad.

- Aceptación de la cruz.

- Docilidad, obediencia...

Aspectos

- Imagen de mí mismo; quién soy yo...

- Actitudes-tendencias para el placer-disfrute, gustos, aficiones.

- Presencia-dominio de ansiedades, miedos, tristezas, angustias, inquietudes...

- El ámbito de la imaginación, dominio de los sentidos...

- Actitud ante las opiniones de los demás, ante los conflictos...

- Medios que usas para crecer en estos aspectos... 


\section{Preguntas}

- ¿Cómo estoy en relación con mi familia?

- ¿Qué cuidado tengo de mi cuerpo (comida, deporte, descanso necesario, tiempo dedicado a la TV, al computador, a Internet)?

- ¿Soy persona feliz, integrada conmigo mismo y con el grupo de amigos, valoro positivamente mis actos?

- ¿Como vivo mi afectividad? ¿Siento que estoy creciendo en el campo de la sexualidad, caminando hacia el amor oblativo?

- ¿Me domina el egoísmo? ¿Estoy centrado y pendiente de mí mismo?

- ¿Relaciones con el otro sexo?

- ¿Me siento amado y feliz en el grupo al que pertenezco?

- ¿Qué dificultades encuentro en mi inserción en la comunidad del seminario?

\section{DIMENSIÓN INTELECTUAL (trabajo-estudio)}

\section{Valores}

- Valorar el estudio como dedicación profesional, como ganar el pan, como justicia social.

- Preocupación por la actualidad social, cultural, eclesial.

- Valorar la formación y no tanto las calificaciones.

- Superar el espíritu competitivo en el estudio.

- Cultivar otras áreas (arte, lenguas, música, literatura...).

- Asumir-valorar los servicios a la comunidad (liturgia, el cocina,

\section{Aspectos} responsabilidades individuales...).

- Horarios de trabajo, de estudio.

- Lecturas complementarias.

- Tiempo dedicado a la TV, al computador, a internet. Sentido, duración, motivos...

- Medios que uso para la preparación intelectual.

\section{Preguntas}

- ¿Cómo estoy en los estudios? ¿Mi actitud en las clases?

- ¿Por qué y para qué estudio? ¿A qué dedico más tiempo, y por qué? ¿Cómo organizo el tiempo de trabajo?

- ¿Cómo preparo las actividades apostólicas que tengo?

- ¿Cuál es mi preocupación por conocer y valorar los problemas del mundo, de la sociedad, de la Iglesia?

- ¿Cuáles son mis lecturas preferidas en los tiempos libres, en vacaciones?

- ¿Qué influencia tienen los estudios en mi crecimiento personal y en mi relación con Dios? 
3. DIMENSIÓN TEOLOGAL (relación con Dios-Cristo-María)

\section{Valores}

- Oración, lectura de la palabra.

- Eucaristía, reconciliación.

- Imagen adecuada de Dios.

- Conocer-amar a Jesucristo.

- Relación con María.

- La Liturgia.

- Descubrir, afianzar, el sentido e historia vocacional.

- Lectura espiritual.

\section{Aspectos}

- El propio proceso vocacional.

- Experiencia de Dios, la búsqueda de Jesús en sí mismo

$y$ en los hombres.

- El rostro de Dios, de Jesús, de María, y la relación con ellos.

- Los porqués de tu deseo de ser sacerdote.

- Sentido eucarístico en tu vida.

- Sentido penitencial, de reconciliación.

- Actitudes de pobreza, de obediencia.

- Comprensión y vivencia de la castidad celibataria.

\section{Preguntas}

- ¿Cómo voy experimentando y viviendo el seguimiento de Jesús en la vida cotidiana?

- ¿Qué experiencia tengo de Jesús resucitado, de la vida nueva en él?

- ¿Qué valor doy a la oración individual, a la lectura de la Palabra y qué tiempos les dedico?

- ¿Cómo es mi oración: meditativa, contemplativa, de alabanza, de súplica? ¿Está presente en ella mi vida, mis relaciones?

- ¿Cómo estoy en cuanto al silencio, la soledad, la interioridad?

- ¿Mi participación en la Eucaristía, en la liturgia de las horas?

- ¿Periodicidad y sentido del sacramento de la reconciliación?

- ¿Importancia y modo de la dirección espiritual?

- ¿Vivencia de las virtudes teologales y de la radicalidad evangélica en pobreza, obediencia, castidad?

- ¿Cómo es la integración de la fe en mi vida?

- ¿Cómo estoy vocacionalmente?

4. DIMENSIÓN COMUNITARIA (relaciones interpersonales)

\section{Valores-Aspectos}

- Capacidad de escucha, de diálogo. 
- Actitud de apertura, de comunicación.

- Aceptación de los otros, de su pensar, de su modo de ser.

- Capacidad-actitud de colaboración, de disponibilidad y de servicio.

- Actitud de compartir, de ayuda.

- Sensibilidad ante las situaciones de los otros, ante sus necesidades.

- Capacidad y ejercicio del perdón.

- Agresividad, conflictividad.

- Actitud positiva, constructiva en el grupo.

- Sinceridad, autenticidad, honradez, respeto.

- Sentido de justicia.

- Sentido y tipo de amistades que cultivo, relaciones con la mujer.

\section{Preguntas}

- ¿Cómo es mi actitud de servicio, de colaboración?

- ¿Cómo es mi contribución a la vida de la comunidad, mi atención a las situaciones y necesidades de los otros, del mundo?

- ¿Hay manifestaciones en mi vida de agresividad, de mentira, de desprecio?

- ¿Soy selectivo en mis relaciones?

- ¿Cómo actúo ante los conflictos, las ofensas?

- ¿Qué tipo y seriedad mantengo en mis relaciones con los otros, con la mujer?

- ¿Cómo pienso vivir mi vida sacerdotal (individualmente, en equipo, en comunidad)?

\section{DIMENSIÓN APOSTÓLICA (actividades pastorales)}

\section{Valores-aspectos}

- Valor formativo de las actividades.

- Valorar la formación teórica.

- La preparación de las actividades.

- Evaluar el desarrollo de las mismas y sus resultados.

- Motivación teológica y pastoral de las actividades.

- La elección de los lugares y destinatarios

- Sensibilidad y aproximación a los pobres, marginados...

- Ejercicio de la responsabilidad y de la autoridad.

\section{Preguntas}

- ¿Como es mi proyección apostólica dentro de la comunidad?

- ¿Mantengo equilibrio entre la oración, el estudio, la actividad apostólica?

- ¿Cuáles son mis motivaciones en las actividades?

- ¿Mis reacciones ante las actividades: feliz, peso, obligación? 
- ¿Cuáles van siendo mis preferencias pastorales y por qué?

- ¿Cuáles son mis experiencias con los pobres, marginados...?

- ¿Reacciones ante las dificultades y los fracasos? 